\title{
Accuracy of staging in primary biliary cirrhosis
}

\author{
M C Garrido, S G Hubscher
}

\begin{abstract}
Aims-To evaluate sampling variability of liver biopsy in patients with primary biliary cirrhosis (PBC).

Methods-Sections from 50 PBC liver specimens obtained at transplantation were examined. The degree of fibrosis was assessed on a scale of $0-4$ using two methods: (1) simulated needle biopsy in fields approximately the size of a conventional needle biopsy; and (2) whole section scanning in areas with little and extensive fibrosis.

Results-Considerable variation in the range of stages of fibrosis was found when the whole section scanning method was used. Only $10(20 \%)$ samples had a consistent degree of fibrosis in all sections scanned. By contrast, the same fibrosis stage was assigned in $30(60 \%)$ specimens examined using the simulated needle biopsy method. When the results obtained by the two methods were compared, there was a discrepancy of one or two stages in 32 samples. This discrepancy was the result of discovering areas with a lesser degree of fibrosis in whole sections compared with the simulated needle biopsy specimens.
\end{abstract}

Conclusion-There is considerable variation in the degree of fibrosis in the livers of patients with PBC, even when end stage specimens obtained at transplantation are examined. Consistent results were obtained when simulated needle biopsy specimens were examined. This, however, may be a reflection of the procedure applied when staging liver needle biopsy specimens, where the greatest degree of abnormality is used in determining the stage. The practice of staging of PBC in small needle biopsy specimens is valuable as long as the appearances are interpreted with caution, bearing in mind that there is considerable variability in the degree of fibrosis.

(F Clin Pathol 1996;49:556-559)

Keywords: primary biliary cirrhosis, stage, accuracy.

Department of Pathology,

The Medical School,

University of

Birmingham,

Edgbaston,

Birmingham B15 2TT

Correspondence to Dr M C Garrido.

Accepted for publication 21 March 1996
Primary biliary cirrhosis (PBC) is a relatively uncommon disorder and mainly affects middle aged women. ${ }^{1}$ It constitutes a progressive liver disease manifested by segmental inflammatory destruction of intrahepatic bile ducts, resulting in progressive loss of these ducts with associated periportal fibrosis and eventually cirrhosis. ${ }^{2}$

Histological staging in PBC is an accepted practice and is thought to be of prognostic value. ${ }^{3}$ Staging is an important part of clinical studies currently carried out in many centres and is used routinely in some of the schemes $\stackrel{\vec{s}}{\rightarrow}$ applied for predicting survival following liver transplantation, ${ }^{4}$ but not in others. ${ }^{5}$

Considerable overlap between stages of PBC $\frac{\bar{c}}{D}$ has been reported. ${ }^{167}$ Rubin et al reported $\stackrel{\mathbb{Q}}{\varrho}$ that the pattern of development of cirrhosis is irregular within the liver in patients with $\mathrm{PBC}{ }^{8} \vec{\circ}$ This situation is potentially misleading when assessing liver biopsy specimens as it might $\vec{\omega}$ introduce an element of sampling error and an 2 individual biopsy sample might not be? representative of the complete histological pic- $\vec{c}$ ture. As all of the studies on sampling variabil- $v$ ity of liver biopsy have included cases of cirrhosis regardless of their aetiology, ${ }^{910}$ we o decided to investigate sampling variability in patients with PBC.

In order to address the problem outlined above, we reexamined sections from a series of $\vec{\varphi}$ 50 livers obtained at transplantation from patients with PBC.

\section{Methods}

We examined the explanted livers from a $\frac{\circ}{\not}$ consecutive series of 50 patients (45 women) $\varrho$ with a diagnosis of PBC admitted to the liver $\stackrel{\circ}{3}$ unit at the Queen Elizabeth Hospital, Birmingham, for liver transplantation between 1991 劳 and 1994. The mean age of the patients was 53.9 years (range 34-68 years). In all patients? the diagnosis of PBC was supported by clinical $\frac{5}{3}$ features, positive anti-mitochondrial antibodies and compatible histological features.

Specimens were sampled according to an established protocol, in which two blocks were obtained from the right and left lobes of the $\frac{D}{0}$ liver. These four blocks were reexamined systematically by one of us (MCG) and the $N$ histological stage recorded on the basis of $N$ appearances on haematoxylin and eosin $\underset{\omega}{N}$ stained slides. For each case one block was also 0 stained with a set of connective tissue stains (Van Gieson, orcein and reticulin). Every section was examined on two separate occa- ? sions: firstly, by means of scanning the whole of $\frac{T}{+}$ the section when two stages were determined $\frac{\mathrm{d}}{\mathrm{D}}$ according to the least and to the most severely affected areas (table 1, range of stage); and sec- $\stackrel{\square}{\square}$ ondly, from every section a needle biopsy specimen sized field (simulated needle biopsy) 8 was examined and staged according to the highest degree of abnormality noted (table 1 , stage in simulated liver biopsy specimen according to lobe). The simulated needle biopsy specimen from each section was obtained by covering the slide with another partly obliterated slide bearing a biopsy specimen sized window $2 \mathrm{~cm}$ in length and $2 \mathrm{~mm}$ in width. 
Table 1 Stage of PBC assigned to each patient by the simulated needle biopsy and whole section scanning methods

\begin{tabular}{|c|c|c|c|c|c|c|c|}
\hline \multirow{2}{*}{$\begin{array}{l}\text { Case } \\
\text { Number }\end{array}$} & \multirow[b]{2}{*}{ Sex } & \multirow{2}{*}{$\begin{array}{l}\text { Age } \\
\text { (years) }\end{array}$} & \multicolumn{4}{|c|}{$\begin{array}{l}\text { Stage in simulated liver biopsy specimens } \\
\text { according to lobe }\end{array}$} & \multirow{2}{*}{$\begin{array}{l}\text { Range of stage } \\
\text { noted on scanning } \\
\text { of whole sections }\end{array}$} \\
\hline & & & Right & Right & Left & Left & \\
\hline 1 & F & 52 & 4 & 4 & 4 & 4 & $4-4$ \\
\hline 2 & $\mathbf{F}$ & 63 & 4 & 4 & 4 & 4 & $3-4$ \\
\hline 3 & $\mathrm{~F}$ & 56 & 4 & 4 & 4 & 4 & $2-4$ \\
\hline 4 & $F$ & 64 & 4 & 4 & 4 & 4 & $4-4$ \\
\hline 5 & $\mathrm{~F}$ & 59 & 4 & 4 & 4 & 4 & $2-4$ \\
\hline 6 & $\mathrm{~F}$ & 46 & 3 & 3 & 2 & 3 & $2-3$ \\
\hline 7 & $\mathrm{~F}$ & 40 & 4 & 4 & 4 & 4 & $3-4$ \\
\hline 8 & $\mathrm{~F}$ & 63 & 4 & 4 & 4 & - & 4-4 \\
\hline 9 & $\mathrm{~F}$ & 59 & 3 & 4 & 4 & 4 & $2-4$ \\
\hline 10 & $\mathrm{M}$ & 53 & 3 & 4 & 4 & 4 & $2-4$ \\
\hline 11 & F & 34 & 3 & 3 & 3 & 3 & $3-3$ \\
\hline 12 & $\mathrm{~F}$ & 61 & 4 & 4 & 4 & 4 & $3-4$ \\
\hline 13 & $\mathrm{~F}$ & 48 & 4 & 4 & $4^{\top}$ & 4 & $3-4$ \\
\hline 14 & $\mathrm{~F}$ & 57 & 4 & - & 3 & 3 & $2-4$ \\
\hline 15 & $\mathrm{~F}$ & 57 & 4 & 4 & 4 & 4 & $2-4$ \\
\hline 16 & $\mathrm{~F}$ & 48 & 3 & 3 & 2 & 2 & $1-3$ \\
\hline 17 & $\mathrm{~F}$ & 53 & 4 & 4 & 4 & 4 & $3-4$ \\
\hline 18 & $\mathrm{~F}$ & 40 & 3 & 3 & 3 & 3 & $2-4$ \\
\hline 19 & $\mathrm{~F}$ & 55 & 4 & 3 & 4 & 4 & $1-4$ \\
\hline 20 & $\mathrm{~F}$ & 68 & 4 & 4 & 4 & 4 & $3-4$ \\
\hline 21 & $F$ & 48 & 3 & 2 & 3 & 3 & $1-4$ \\
\hline 22 & $\mathrm{~F}$ & 42 & 4 & 4 & 4 & 4 & $4-4$ \\
\hline 23 & $\mathrm{~F}$ & 61 & 4 & 4 & 4 & 4 & $4-4$ \\
\hline 24 & $\mathrm{~F}$ & 67 & 3 & 3 & 4 & 4 & $2-4$ \\
\hline 25 & $\mathrm{~F}$ & 51 & - & 3 & 3 & 4 & $3-4$ \\
\hline 26 & $\mathrm{~F}$ & 54 & 4 & 4 & 4 & 4 & $4-4$ \\
\hline 27 & $\mathrm{~F}$ & 61 & 4 & 4 & 4 & 4 & $4-4$ \\
\hline 28 & $\mathrm{~F}$ & 52 & 2 & 2 & 2 & - & $2-2$ \\
\hline 29 & $\mathrm{~F}$ & 62 & 4 & 4 & 4 & 4 & $2-4$ \\
\hline 30 & $\mathrm{~F}$ & 59 & 4 & 3 & 4 & 3 & $2-4$ \\
\hline 31 & $\mathrm{~F}$ & 41 & 3 & 3 & 4 & 3 & $3-4$ \\
\hline 32 & $\mathrm{M}$ & 55 & 4 & 4 & 4 & 4 & $3-4$ \\
\hline 33 & $\mathrm{~F}$ & 64 & 4 & 4 & 4 & 4 & $3-4$ \\
\hline 34 & $\mathrm{~F}$ & 61 & 4 & 4 & 4 & 4 & $3-4$ \\
\hline 35 & $\mathbf{M}$ & 37 & 4 & 4 & 4 & 4 & $3-4$ \\
\hline 36 & $\mathrm{~F}$ & 45 & 4 & 4 & 4 & 4 & $3-4$ \\
\hline 37 & $\mathrm{~F}$ & 55 & 2 & 2 & 3 & 2 & $2-4$ \\
\hline 38 & $\mathrm{~F}$ & 68 & 4 & 4 & 4 & 4 & $3-4$ \\
\hline 39 & $\mathbf{M}$ & 49 & 4 & 3 & 4 & 4 & $3-4$ \\
\hline 40 & $\mathrm{~F}$ & 42 & 2 & 2 & 2 & 2 & $1-3$ \\
\hline 41 & $\mathrm{~F}$ & 60 & 4 & 4 & 4 & 4 & $3-4$ \\
\hline 42 & $\mathrm{M}$ & 46 & 4 & 3 & 4 & 4 & $1-4$ \\
\hline 43 & $\mathrm{~F}$ & 53 & 4 & 4 & 4 & 4 & $3-4$ \\
\hline 44 & $\mathrm{~F}$ & 64 & 4 & 4 & 4 & 4 & $3-4$ \\
\hline 45 & $\mathrm{~F}$ & 65 & 3 & 4 & 3 & 4 & $3-4$ \\
\hline 46 & $\mathrm{~F}$ & 59 & 4 & 4 & 4 & 4 & $4-4$ \\
\hline 47 & $\mathrm{~F}$ & 39 & 3 & 3 & 3 & 2 & $2-3$ \\
\hline 48 & $\mathrm{~F}$ & 58 & 3 & 3 & 3 & 4 & $2-4$ \\
\hline 49 & $\mathrm{~F}$ & 55 & 3 & 3 & 4 & 4 & $3-4$ \\
\hline 50 & $\mathrm{~F}$ & 47 & 3 & 3 & 4 & 4 & $3-4$ \\
\hline
\end{tabular}

Scoring system: stage 1 = no fibrosis; stage 2 = periportal fibrosis (fibrous expansion of porta tracts without bridging); stage $3=$ bridging fibrosis (portal-portal or portal-central linkage); stage 4 = established cirrhosis. the 50 cases studied, 44 had areas of established cirrhosis (stage 4 ); the remaining six cases showed variable fibrosis without true regenerative nodules seen anywhere in the liver. Five of these had stage 3 fibrosis and the other stage 2 , which showed conspicuous bile duct loss. When the range of severity of fibrosis (range of stages) was analysed (table 1), only 10 cases had a consistent degree of fibrosis throughout all of the sections scanned. Of these, eight cases were assigned stage 4 fibrosis in all sections, one case stage 3 and another stage 2 . In all the remaining 40 cases, scanning showed variable degrees of fibrosis, with 23 cases showing variation by one stage (for example, 3-4, 2-3), 14 cases showing variation by two stages (for example, 1-3, 2-4) and three cases by three stages (for example, 1-4).

ASSESSMENT OF INDIVIDUAL CASES BY SIMULATED NEEDLE BIOPSY

More consistent results were generally obtained when individual cases were analysed with the simulated needle biopsy method. Forty one cases had at least one biopsy specimen which showed established cirrhosis (stage 4); the remaining nine cases showed variable fibrosis not amounting to cirrhosis. Of these, seven were stage 3 and two were stage 2 . Analysis of the range of stages assigned using the simulated needle biopsy method showed that of the 50 cases examined, 31 showed the same stage in all four specimens examined. Of these 27 cases were stage 4 , two cases were stage 3 and two were stage 2 . In the remaining 19 cases there was variation of only one stage (for example, 3-4, 2-3).

COMPARISON OF STAGES ASSIGNED BY THE TWO METHODS OF EXAMINATION

No overall discrepancy was found in 18 cases $(36 \%)$ when the ranges of stages, as determined by the two methods, were compared. Eight cases showed stage 4 fibrosis in all the fields examined; six showed changes ranging between stages 3 and 4; two showed changes ranging between stages 2 and 3; one case was stage 3 ; and another stage 2 by both methods.

In the remaining $32(64 \%)$ cases, where a discrepancy in the stages assigned was found, there was a difference of one stage for 26 $(52 \%)$ cases and of two stages for six (12\%). Table 1 shows that when the whole of the section was scanned, 22 of a total 26 cases showing a discrepancy of one stage, contained areas with a lesser degree of fibrosis than that seen when the sections were examined by the simulated needle biopsy method; three cases showed both a higher and lower degree of abnormality, as determined on scanning, compared with the stages obtained with the simulated needle biopsy method (cases 18, 21 and 40). In addition, one case showed areas with a higher degree of fibrosis on scanning than on the simulated needle biopsy method (case 37). The discrepancy of two degrees of severity found in six cases (cases $3,5,15,19$, 29 , and 42) was the result of having found areas of liver showing a much lesser degree of fibrosis by scanning the whole of the sections
SECTION SCANNING

When individual cases were analysed, notable discrepancy was seen frequently when the whole section scanning method was used. Of 
than were found by the needle biopsy method. Of these, four cases (cases 3, 5, 15, and 29) were stage 4 on simulated needle biopsy and stages as low as 2 were assigned to them by scanning. The remaining two cases were stage 3 or 4 on simulated needle biopsy but also had areas of stage 1 fibrosis (cases 19 and 42). None of the cases showed two higher degrees of abnormality when whole sections were scanned than when examined by simulated needle biopsy.

In summary, 28 of the 32 cases where there was a discrepancy had areas of less severe fibrosis when the section was examined as a whole; six of these showed a discrepancy of two stages. Only one case had areas with a higher degree of fibrosis and two cases had areas of both higher and lower degrees of abnormality on scanning than on simulated needle biopsy. Overall, there were no significant differences in the stages assigned with respect to the right and left lobes of the liver with either method.

\section{Discussion}

When one compares the four different staging methods for PBC currently in use, ${ }^{2811} 12$ they are roughly comparable, but each may be difficult to apply. For all staging systems, the initial lesion is portal in location, with destruction of small bile ducts. This is referred to as the nonsuppurative destructive cholangitis or portal hepatitis by some authors, ${ }^{2}$ or as the florid duct lesion $^{11}$ or cholangitis by others. ${ }^{12}$

Progression in PBC takes the form of increasing fibrosis, initially limited to portal tracts. This is followed by extension from portal areas into the adjacent liver parenchyma by two main possible mechanisms: (1) lymphocytic piecemeal necrosis giving rise to appearances not unlike those of chronic active hepatitis; or (2) bile ductular proliferation and biliary piecemeal necrosis which may result, in part, from the effects of cholate stasis in periportal hepatocytes. Different staging systems have different staging criteria. Whereas Scheuer and Popper ${ }^{11}{ }^{12}$ rely on ductular proliferation as the criterion for defining stage 2 PBC, Ludwig's system ${ }^{2}$ uses periportal hepatitis as the feature defining stage 2 .

In trying to apply the above staging systems to needle biopsy specimens, we have noted that expanded portal tracts may show only one of the two main patterns of damage - that is, ductular proliferation or periportal hepatitis. Indeed, in some cases, septa may fail to show conspicuous ductular proliferation or lymphocytic piecemeal necrosis, whereas in others both patterns of damage may be seen at the same time.

For this reason in the present study we decided to apply a simplified staging system that was practical and easy to use. This system relies on the presence of fibrosis and its degree of severity, regardless of accompanying ductular proliferation or the degree of inflammation. This simplified staging system basically represents an amalgamation of the systems referred to earlier and is in line with concepts applied more recently for staging fibrosis in other forms of chronic liver disease. ${ }^{13}$
The results of the present study demonstrate that when applying the whole section scanning method of examination, to sections obtained from explanted livers from patients with PBC, notable discrepancy in the range of severity is $c$ seen frequently. Only 10 cases $(20 \%)$ showed a $\Omega$ consistent degree of fibrosis throughout the sections scanned. These observations give support to the concept of the patchy nature of $\overrightarrow{\vec{\sigma}}$ development and progression of fibrosis in PBC. Forty four cases had areas consistent $\overrightarrow{\vec{D}}$ with established cirrhosis, a finding which is $\overrightarrow{0}$ not surprising given the fact that the patients studied were candidates for transplantation. $\frac{\overline{\bar{p}}}{\bar{\partial}}$ Rather more surprisingly, however, in six of the $\mathbb{Q}$ 50 patients studied, there was no evidence of true cirrhosis in any of the sections examined $\vec{\sim}$ and in one of these there were no areas exceed- ? ing stage 2 fibrosis. In this particular case there $\overrightarrow{\vec{\omega}}$ was conspicuous bile duct loss throughout.

More consistent results were obtained when $\frac{8}{0}$ the simulated needle biopsy method was applied. In $31(62 \%)$ patients the degree of $-v$ severity of fibrosis found was consistent in all of simulated needle biopsy specimens examined; $ᄋ$ of these, the majority (27/31) were stage 4 을 fibrosis. As for the overall degree of severity of $\vec{c}$ fibrosis found in cases examined with this method, a large proportion (41 cases) had $\overrightarrow{0}$ established cirrhosis.

Some of the differences observed when the two methods of assessing the sections were compared may simply be a reflection of the procedure applied for staging liver needle biopsy specimens, where the greatest degree of abnormality noted in the specimen is taken into account when determining the stage. This could account for the greater degree of consistency and generally higher stages noted using the simulated needle biopsy method. However, scanning whole sections suggested that needle biopsy assessments may considerably overesti- $\frac{0}{3}$ mate the degree of fibrosis. Of the 50 cases, 28 had lesser degrees of fibrosis in whole sections compared with simulated needle biopsy specimens and six of these had areas of fibrosis two stages in severity lower than those observed in $\frac{D}{2}$ the latter. Conversely, an area with a higher degree of abnormality was seen in one case $\widetilde{N}$ only on whole section scanning than when they $N$ were staged using the simulated needle liver biopsy method.

It has been considered an advantage that some models for predicting survival following $\frac{C}{\Phi}$ liver transplantation are based on clinical vari- ? ables only and are independent of histological 0 staging. ${ }^{5}$ The results of the present study $\frac{\vec{D}}{\mathbb{D}}$ support the view that the results of histological staging should be used with caution in predictive models of this sort.

Overall, these results give support to the $\delta$ variability in the pattern of evolution of PBC as reported by some $e^{167}$ and the irregular pattern of development of cirrhosis in $\mathrm{PBC}^{8}$ as reported by others, a finding which, to the best of our knowledge, had never been demonstrated formally. These results are in agreement with those of previous studies looking at sampling variability of percutaneous liver biopsy in patients with cirrhosis, regardless of 
the aetiology of their condition. ${ }^{9}{ }^{10}$ Furthermore, the results in this study are in keeping with the results published recently by Olsson $e t$ $a l^{14}$ when similar observations were made on a series of liver biopsy specimens from patients with primary sclerosing cholangitis.

The practice of staging of PBC in small needle biopsy specimens is valuable as long as the appearances in these specimens are interpreted with caution, bearing in mind that there is considerable variability in stage. The results of this study suggest that stages obtained by examining needle biopsy specimens may overestimate the degree of fibrosis in the liver.

We are grateful to our colleagues on the Liver Unit, Queen Elizabeth Hospital, Birmingham (Dr D H Adams, Mr J A C Buckels, Dr E Elias, Mr A D Mayer, Mr P Mc Master, Dr D J Mutimer, and Dr J M Neuberger), for providing us with the opportunity to study material from patients under their care.

1 Portmann B, MacSween RNM. Diseases of the intrahepatic bile ducts. In: MacSween RNM, Anthony PP, Scheuer PJ, eds. Pathology of the liver. Edinburgh: Churchill Livingstone, 1987:424

2 Ludwig J, Dickson ER, McDonald GSA. Staging of chronic nonsuppurative destructive cholangitis (syndrome of primary biliary cirrhosis). Virchows Arch A Pathol Anat Histopathol 1978;379:103-12.

3 Roll J, Boyer JL, Barry D, Klatskin G. The prognostic importance of clinical and histological features in asymp- tomatic and symptomatic primary biliary cirrhosis. $N$ Engl f Med 1983;308:1-7.

4 Neuberger JM, Gunson BK, Buckels JAC, Elias E, McMaster P. Referral of patients with primary biliary cirrhosis for liver transplantation. Gut 1990;31:1069-72.

5 Grambsch PM, Dickson ER, Wiesner RH, Langworthy A. Application of the Mayo primary biliary cirrhosis survival model to Mayo liver transplant patients. Mayo Clin Proc 1989;64:699-704.

6 Scheuer PJ. Biliary disease. In: Scheuer PJ, Lefkowitch JH, eds. Liver biopsy interpretation. Philadelphia:WB Saunders, 1994:51.

7 Wight DGD. Cholestasis and intrahepatic bile ducts. In: Symmers WStC, Wight DGD, eds. Systemic pathology. Liver, biliary tract and exocrine pancreas. Vol 11. Edinburgh Churchill Livingstone, 1994:306.

8 Rubin E, Schaffner F, Popper H. Primary biliary cirrhosis. Chronic non-suppurative destructive cholangitis. $\mathrm{Am} \mathcal{F}$ Pathol 1965;46:387-407.

9 Abdi W, Millan JC, Mezey E. Sampling variability on percutaneous liver biopsy. Arch Intern Med 1979;139:667-9.

10 Maharaj B, Leary WP, Naran AD, Maharaj RJ, Cooppan RM, Pirie D. Sampling variability and its influence on the diagnostic yield of percutaneous needle biopsy of the liver. Lancet 1986;i:523-5.

11 Scheuer PJ. Primary biliary cirrhosis. Proc R Soc Med 1967; 60: $1257-60$.

12 Popper H, Schaffner F (eds). Non-suppurative destructive chronic cholangitis and chronic hepatitis. In: Progress in liver diseases. Vol III. New York: Grune \& Stratton, 1970:336-54.

13 Scheuer PJ. Classification of chronic viral hepatitis: a need for reassessment. F Hepatol 1991;13:372-4.

14 Olsson R, Hägerstrand I, Broomé U, Danielsson Å, Järnerot G, Lööf $\mathrm{L}$ et al. Sampling variability of percutaneous liver biopsy in primary sclerosing cholangitis. $\mathcal{f}$ Clin Pathol 1995;48:933-5. 